\title{
META ANALYSES OF CORRELATED MULTIPLE BASELINE TIME SERIES DESIGN INTERVENTION MODELS USING JR ESTIMATE
}

\section{OLUWAGBOHUNMI AWOSOGA ${ }^{1}$, JOSEPH MCKEAN ${ }^{2}$ and BRADLEY HUITEMA ${ }^{3}$}

${ }^{1}$ Faculty of Health Science

University of Lethbridge

Lethbridge, Alberta

Canada

e-mail: olu.awosoga@uleth.ca

${ }^{2}$ Department of Statistics

Western Michigan University

Kalamazoo

Michigan 49008-5152

USA

${ }^{3}$ Department of Psychology

Western Michigan University

Kalamazoo, Michigan

USA

\begin{abstract}
This study develops $\mathrm{R}$ estimators of the fixed effects in an experiment done over correlated baseline series. Besides a simple parametric approach we investigate linear mixed models procedures also. The random errors are independent within 2010 Mathematics Subject Classification: 97K70.

Keywords and phrases: joint rank, meta-analysis, robustness, multiple baseline, correlated tests, weighted Wilcoxon, contaminated normal distribution.

Received June 30, 2016
\end{abstract}

(C) 2016 Scientific Advances Publishers 
series but are dependent between several baseline series. The JR method seems more appropriate in term of its empirical type I error and the power of the test for the case of independence within but dependence between series than other methods (i.e., CT, WW, and LME) considered in this study. We illustrated the robustness of the procedures on a real data set which contained some outliers. Our robust procedures were much less sensitive to the effect of the outliers than the traditional analysis based on LS. A simulation study over situations similar to that of the data set confirmed the validity of our new approaches. The study also showed the robustness of efficiency of our approach over that of the traditional analysis.

\section{Introduction}

In this study, we develop $R$ estimators of the fixed effects in an experiment done over correlated baseline series. Besides a simple parametric approach we investigate linear mixed models procedures also. The case of independence within series but dependence between series are common in repeated measure designs, split plot designs, multi-center clinical trials, and so on. The random errors are independent within series but are dependent between several baseline series. The dependence is at the time (or day) of recording. It seems reasonable to capture this dependence with a random effect. Hence, we have the case of a simple mixed model. We investigate two procedures. The classical procedure based on normal likelihood theory and a robust $\mathrm{R}$ procedure recently proposed by Kloke et al. [13]. The asymptotic theory of the estimators is similar to that of $\mathrm{R}$ estimators for linear model where all random errors are independent of one another.

\section{Methodology}

This section gives a brief description of each method adopted in this study with specific model and their assumptions.

\subsection{The correlation test (CT)}

This simple parametric procedure was initiated by Huitema et al. [9] to obtain the overall $z$-test statistic for correlated series. It is a parametric method which uses the least squares procedure to generate 
the correlation test statistic. This approach starts out by first obtaining a correlation matrix for the multiple baseline series, a $t$-test statistic for each of the series, and then combining these in the "usual" way for the test statistic. As aforementioned, if the baseline series are not independent (correlated between series), then the overall $z$-test statistic for the LC (or SC) is computed as follows:

$$
t=\frac{J^{T} t_{i}}{\sqrt{J^{T} R J}},
$$

where $R$ is the pooled within phase correlation matrix of all series; $J$ is an $m$ by 1 vector of ones; and $t_{i}$ is the corresponding test statistic for each baseline series.

$d f=N-2 \times$ (Number of baseline series).

We also obtain the corresponding $p$-value and the critical value for this correlated $t$ test statistic based on the assumption that $t$ follows a $t$-distribution. We compare the result with other methods such JR, LME, and WW in our simulation report given in the later part of this study.

\subsection{Linear mixed-effects (LME)}

Mixed-effects models provide a flexible and powerful tool for the analysis of grouped data in which the response variable is (at least approximately) continuous while both the random effect and the errors follow Gaussian distributions. Let $Y_{i j}$ denote the response for the time point $i$ and series $j . Y_{1 j}$ is an $m \times 1$ vector of responses, $X_{i j}$ is $m \times p$ design matrix, and $e_{1 j}$ is the $m \times 1$ vector of errors. Suppose $\mathbf{1}_{\mathbf{m}}$ denote a vector of $m$ ones, then our model for correlated baseline series would be modified as

$$
Y_{i j}=\mathbf{X}_{\mathrm{ij}}^{\prime} \beta+b_{i}+\epsilon_{i j}, \quad j=1, \ldots, n ; i=1, \ldots, m,
$$

where $\beta$ is the vector of regression coefficients, $b_{i}$ is the random effect for time point $i$ and $e_{i j}$ is the vector of independent random errors. Note that for each series $j$, we model the error vector $e_{j}$ as 


$$
e_{j}=\mathbf{1}_{\mathbf{m}} b_{j}+\epsilon
$$

where the components of $\epsilon$ are independent and identically distributed and $b_{j}$ is a continuous random variable which is independent of $\epsilon$. Also, assume that the random effects $b_{1}, b_{2}, \ldots, b_{j}$ are independent and identically distributed random variables.

A model with both fixed effects and random effects is called a mixedeffects model. For between series, we have $b_{i} \sim N\left(0, \sigma_{b}^{2}\right)$ and for within series, $\epsilon_{i j} \sim N\left(0, \sigma^{2}\right)$. The covariance between observations in the same series is $\sigma_{b}^{2}$ corresponding to a correlation of $\frac{\sigma_{b}^{2}}{\sigma^{2}+\sigma_{b}^{2}}$ assuming multivariate normality. The LME function from the nlme library for $\mathrm{R}$ is used for fitting the linear mixed-effects model using, maximum likelihood (ML).

A randomized block design is a type of experiment in which there are two classification factors: An experimental factor for which we use fixed effects and a blocking factor for which we use random effects. Some of the terms in LME output include: REML (restricted maximum likelihood), AIC (Akaike information criterion), and BIC (Bayesian information criterion or SBC - Schwarz Bayesian criterion); where AIC $=-2 \log l i k+$ 2npar and $\mathrm{BIC}=-2 \log l i k+n p a r \log (N)$. (See Sakamoto et al. [16] and Schwarz [17]).

The confidence intervals on the variance components should be checked after fitting a model. Having abnormally wide intervals usually indicates problems with the model definition.

\subsection{JR estimate}

An extension of the $R$ estimators based on the joint ranks (JR) of all residuals developed by Kloke et al. [13] to estimate the fixed effects in a linear model with cluster correlated continuous error distributions for general score functions is adopted in this section with some modifications 
to suit correlated multiple baseline time-series design. The random errors are dependent between different series but are independent within each series.

In this study, we use an arbitrary score function, thus the estimator can be optimized if information is available on the underlying distribution of the errors. For a simple mixed model with block (i.e., each baseline series) as a random effect, consider an experiment conducted over $p$ series, where series $j$ has $m$ observations. We adopt the same model as discussed in LME above. The procedure is asymptotically distribution free. In particular, no assumption of normality is required. In terms of the procedure proposed by Kloke et al. [13], the $m$ clusters are the time points. The estimate of $\beta$ is the JR-estimate which we denote as $\hat{\beta}_{\varphi}$.

The asymptotic variance-covariance matrix of $\hat{\beta}_{\varphi}$ simplifies to,

$$
V_{\varphi}\left(\underline{\beta}_{R}\right)=\underline{\tau}_{\varphi}^{2}\left(X^{\prime} X\right)^{-1}\left(\sum_{i}^{n} X_{i}^{\prime} \sum_{\varphi, i} X_{i}\right)\left(X^{\prime} X\right)^{-1}
$$

where

$$
\sum_{\varphi, i}=\left(1-\rho_{\varphi}\right) I_{n_{i}}+\rho_{\varphi} J_{n_{i}}
$$

with a scale parameter

$$
\tau_{\varphi}^{-1}=\int_{0}^{1} \varphi(u)\left\{\frac{-f^{\prime}\left[F^{-1}(u)\right]}{f\left[F^{-1}(u)\right]}\right\} d u,
$$

and

$$
\rho_{\varphi}=\operatorname{cov}\left\{\varphi\left[F\left(e_{11}\right)\right], \varphi\left[F\left(e_{12}\right)\right]\right\}=E\left\{\varphi\left[F\left(e_{11}\right)\right], \varphi\left[F\left(e_{12}\right)\right]\right\}
$$

\{see Kloke et al. [13]\}. 
If we assume that the variances of the errors exist (say $\sum_{e_{j}}$ ), then the variance-covariance matrix of $e_{j}$ is compound symmetric having the form $\sum_{e_{j}}=\sigma^{2} A_{j}(\rho)=\sigma^{2}\left[(1-\rho) I_{m}+\rho J_{m}\right]$, where $\sigma^{2}=\operatorname{var}\left(e_{j k}\right), I_{m}$ is the identity matrix of order $m$, and $J_{m}$ is an $m \times m$ matrix of ones. Note that $\sigma^{2}=\sigma_{\epsilon}^{2}+\sigma_{b}^{2}$, hence, $\rho=\frac{\sigma_{b}^{2}}{\sigma_{\epsilon}^{2}+\sigma_{b}^{2}}$. We refer to these parameters $\left(\sigma_{\epsilon}^{2}, \sigma_{b}^{2}, \sigma^{2}\right)$ as the variance components. We do not need estimates of these variance components for our inference.

The values for JR and LME test statistic were obtained based on the following analogy: Suppose $V(\hat{\eta})=\hat{\sum}_{\eta}$, where $\hat{\eta}$ is the parameter estimate. Recall if $A=\Gamma D \Gamma^{\prime}$, then

$$
A^{-\frac{1}{2}}=\Gamma D^{-\frac{1}{2}} \Gamma^{\prime} . \text { Thus } \hat{\varphi}=\hat{\sum}_{\eta}^{-\frac{1}{2}} \hat{\eta} \Rightarrow V(\hat{\phi})=\hat{\sum}_{\eta}^{-\frac{1}{2}} \hat{\sum}_{\eta} \hat{\sum}_{\eta}^{-\frac{1}{2}}=1
$$

This shows that they are stochastically independent, hence we used a sort of multivariate analysis approach (in the form of Hotelling $T^{2}$ ) to obtain the test statistic for the JR and LME meta-analytic representation as

$$
t=\frac{1^{\prime} \hat{\psi}}{\sqrt{3}}
$$

The following is a design matrix used for multiple baseline model based on both JR and LME approach. It is 3-series specific. 
Table 1. Special design matrix for multiple baseline time series intervention model (JR and LME)

\begin{tabular}{|c|c|c|c|c|c|c|c|c|c|c|c|}
\hline$\beta_{11}$ & $\beta_{12}$ & $\beta_{13}$ & $\beta_{14}$ & $\beta_{21}$ & $\beta_{22}$ & $\beta_{23}$ & $\beta_{24}$ & $\beta_{31}$ & $\beta_{32}$ & $\beta_{33}$ & $\beta_{34}$ \\
\hline 1 & 1 & 0 & 0 & 0 & 0 & 0 & 0 & 0 & 0 & 0 & 0 \\
\hline 1 & 2 & 0 & 0 & 0 & 0 & 0 & 0 & 0 & 0 & 0 & 0 \\
\hline . & & . & . & & . & & & . & . & . & \\
\hline . & . & . & . & & . & . & & & . & . & \\
\hline . & . & . & . & & & . & & & & & \\
\hline 1 & $n_{1}$ & 0 & 0 & 0 & 0 & 0 & 0 & 0 & 0 & 0 & 0 \\
\hline 1 & $n_{1}+1$ & 1 & 0 & 0 & 0 & 0 & 0 & 0 & 0 & 0 & 0 \\
\hline 1 & & 1 & 1 & 0 & 0 & 0 & 0 & 0 & 0 & 0 & 0 \\
\hline 1 & & 1 & 2 & 0 & 0 & 0 & 0 & 0 & 0 & 0 & 0 \\
\hline & & . & . & . & . & & . & & . & $\cdot$ & . \\
\hline 1 & $n$ & 1 & $n_{2}-1$ & 0 & 0 & 0 & 0 & 0 & 0 & 0 & 0 \\
\hline 1 & 1 & 0 & 0 & 1 & 1 & 0 & 0 & 0 & 0 & 0 & 0 \\
\hline 1 & & 0 & 0 & 1 & & 0 & 0 & 0 & 0 & 0 & 0 \\
\hline 1 & & 0 & 0 & . & & 0 & 0 & 0 & 0 & 0 & 0 \\
\hline 1 & . & 0 & 0 & & . & 0 & 0 & 0 & 0 & 0 & 0 \\
\hline 1 & $n_{1}$ & 0 & 0 & . & $n_{1}$ & 0 & 0 & 0 & 0 & 0 & 0 \\
\hline 1 & $n_{1}+1$ & 0 & 0 & 1 & $n_{1}+1$ & 1 & 0 & 0 & 0 & 0 & 0 \\
\hline 1 & & 0 & 0 & 1 & . & & 1 & 0 & 0 & 0 & 0 \\
\hline 1 & & 0 & 0 & . & . & & 2 & 0 & 0 & 0 & 0 \\
\hline 1 & & 0 & 0 & . & . & & . & 0 & 0 & 0 & 0 \\
\hline 1 & $n$ & 0 & 0 & 1 & $n$ & 1 & $n_{2}-1$ & 0 & 0 & 0 & 0 \\
\hline 1 & 1 & 0 & 0 & 0 & 0 & 0 & 0 & 1 & 1 & 0 & 0 \\
\hline 1 & & 0 & 0 & 0 & 0 & 0 & 0 & $\cdot$ & . & 0 & 0 \\
\hline 1 & & 0 & 0 & 0 & 0 & 0 & 0 & . & . & . & . \\
\hline 1 & & 0 & 0 & 0 & 0 & 0 & 0 & . & . & . & . \\
\hline 1 & $n_{1}$ & 0 & 0 & 0 & 0 & 0 & 0 & 1 & $n_{1}$ & 0 & 0 \\
\hline
\end{tabular}


Table 1. (Continued)

\begin{tabular}{cccccccccccc}
\hline 1 & $n_{1}+1$ & 0 & 0 & 0 & 0 & 0 & 0 & 1 & $n_{1}+1$ & 1 & 0 \\
1 &. & 0 & 0 & 0 & 0 & 0 & 0 &. &. &. & 1 \\
1 &. & 0 & 0 & 0 & 0 & 0 & 0 &. &. &. &. \\
1 &. & 0 & 0 & 0 & 0 & 0 & 0 &. &. &. &. \\
1 & $n$ & 0 & 0 & 0 & 0 & 0 & 0 & 1 & $n$ & 1 & $n_{2}-1$ \\
\hline
\end{tabular}

Where $j$ is the number of parameters, $i=1,2, \ldots, p ; j=1,2,3,4 ; p=3$ (i.e., number of baseline series); $n_{1}$ is the number of pre-intervention observations, $n_{2}$ is the number of post-intervention observations and $n=n_{1}+n_{2}$ is the total observations in each series.

\section{Monte Carlo Evaluation}

This was designed to evaluate the performance of these new tests with respect to adequacy of type I error and power of the test and competitors over a variety of distributions. In this section, we considered the competing procedures discussed in the case of independence within series but dependence between series. Some 15000 simulations were generated. Empirical type I error rate and power functions were investigated. The four-parameter intervention model described in Awosoga [1] was specified with parameter values: $\beta_{0}=0, \beta_{1}=0, \beta_{2}=0$, and $\beta_{3}=0$. Furthermore, intervention points were specified to occur at halfway through each simulation process, regardless of the total number of observations in the simulated series. For the null model, we chose the following distributions: normal; contaminated normal (with $P=0.10$, 0.20 and $\sigma_{c}=10$ and 25, respectively); Cauchy; and contaminated skewed Cauchy distributions (with $\mu=30, P=0.10,0.20$ and $\sigma_{c}=10$ and 25 , respectively). 
Let $\mathbf{Y}=\left(Y_{1}, \ldots, Y_{n}\right)^{\prime}$ denote $n \times 1$ vector of observations and let $\mathbf{X}$ denote the $n \times p$ matrix whose $i$-th row is $x_{i}^{\prime}$. We can then express our model for case of dependence within series, but independence between two or more baselines and case of dependence within series, and between two or more baselines as

$$
\mathbf{Y}_{t}=\mathbf{1} \beta_{0}+X \beta+\epsilon_{t}
$$

where 1 is an $n \times 1$ vector of ones and the error structure associated with our model is a first-order autoregressive process defined as

$$
\epsilon_{t}=\rho \epsilon_{t-1}+a_{t},
$$

where $\epsilon_{t}$ is the process error at time $t ; \rho$ is the lag-1 autocorrelation of the $\epsilon_{t}$; and $a_{t}$ is the random shock meeting the assumption of constant variance, normality and independence. Each error series was started up with a standard normal variate. For each specified value of $\rho$ and series length $N, 15000$ interrupted series were generated. The phase lengths were equal $\left(n_{1}=n_{2}\right)$. The $a_{t}$ s were generated as independent normal deviates from a pair of independent uniform variates as proposed by Marsaglia and Bray [14]. Since the model include intercept parameter, $\beta_{0}$, there is no loss of generality in assuming that $\mathbf{X}$ is centered; i.e., the columns of $\mathbf{X}$ sum to 0 . Note that the error structure associated with our model is estimated by

$$
\hat{\epsilon}_{t}=\rho \hat{\epsilon}_{t-1}+a_{t}
$$

which was used as the first-order autoregressive process error.

A contaminated normal random variable is of the form

$$
H=\left(1-I_{\epsilon}\right) Z+I_{\epsilon} \sigma_{c} Z
$$

where $I_{\epsilon}$ and $Z$ are independent random variables, $Z$ has a standard normal distribution, and $I_{\epsilon}$ is either 1 or 0 with probability $\epsilon$ or $1-\epsilon$, 
respectively. While a contaminated skewed Cauchy random variable has the form

$$
G=\left(1-I_{\epsilon}\right) W+I_{\epsilon}\left[\sigma_{c} W+\mu\right],
$$

where $I_{\epsilon}$ is a binomial random variable which takes on values 0 or 1 with probability $1-\epsilon$ or $\epsilon$, respectively, and $W$ is a Cauchy random variable which is independent of $I_{1-\epsilon}$.

The two major criteria for the evaluation of an inferential test are (1) validity (adequacy of type I error performance) and (2) power. A Monte Carlo simulation experiment was designed to evaluate the performance of the new robust test with respect to these criteria. For the situations where the code is in Fortran, the uniform variates were generated by the portable Fortran generator UNI developed by Kahner et al. [10]. For others, the first simulated autoregressive series $\left\{\epsilon_{t}\right\}_{i}$ was started after the generation of 300 variates of the form $u_{j}=\rho u_{j-1}+e_{j}$, where $j=1, \ldots, 300$. Letting $\epsilon_{0}=u_{300}$, the first simulated autoregressive series $\left\{\epsilon_{t}\right\}_{i}$ was generated as $\epsilon_{i}=\rho \epsilon_{i-1}+a_{i}$, where $i=1, \ldots, n$. Then this entire process was repeated for each of the 15000 simulations. Hence, between each generated series 300 ignored variates were generated. Thus, each simulated series began with a random normal

deviate with $\mu=0, \sigma^{2}=\frac{1}{\left(1-\rho^{2}\right)}$, and this gives an assurance that the last observation of one simulated series was virtually uncorrelated with the first observation of the next simulated series.

\section{Comparison of Methods using $N=30$ with Simulation Size $=15000$}

Four methods were compared under the case of independence within series, but dependence between two or more baselines and Case of dependence within series, and between two or more baselines in this 
section. We adopt same model as discussed in LME above. The procedure is asymptotically distribution free. The methods involved are joint rank (JR) estimation, linear mixed effect (LME) model, correlation test (CT), and weighted Wilcoxon (WW) estimate. For each simulation, we used the same design matrix as shown in Appendix A (but with some modification for JR and LME) which were three series specific (see JR section). Thus, some modification would be required to adapt our codes to multiple baseline time series design intervention models involving more than three series. For independence within series, but dependence between two or more baselines, we used the model

$$
Y_{i j}=X_{i j}^{\prime} \beta+b_{i}+e_{i j}, \quad j=1,2, \ldots, n ; i=1,2, \ldots, m,
$$

where the random effects $b_{1}, b_{2}, \ldots, b_{m}$ are independent and identically distributed, and are independent of the errors $e_{i j}$. For the simulation study, the distribution of the random effects is $b_{i} \sim N\left(0, \sigma_{b}^{2}\right)$. The variance of the random effect was set at $\sigma_{b}^{2}=4$. Since the variance of $e_{i j}$ was set at 1 , this implies that the intraclass corrections for the study is $\rho=\frac{\sigma_{b}^{2}}{\sigma^{2}+\sigma_{b}^{2}}=\frac{4}{4+1}=0.80$. We focused our discussion on level-change (LC) and slope-change (SC). The following tables obtained from 15000 simulations exhibit the empirical alpha level, empirical type I error and the power study for these tests under four distributions with varied LC and SC.

\subsection{Cases of normal error with 15000 simulations}

Table 2. Summary table for the empirical alpha level with normal errors (four parameter model)

\begin{tabular}{|c|c|c|c|c|}
\hline$N=30$ & JR & LME & CT & WW \\
\hline LC (Null) & 0.0411 & 0.0616 & 0.0436 & 0.0314 \\
\hline SC (Null) & 0.0453 & 0.0661 & 0.0465 & 0.0347 \\
\hline
\end{tabular}


Comment: It is clearly seen that WW is more conservative while LME is liberal. JR and CT look good.

Table 3. Summary table for the power $(\mathrm{LC}=8)$ with normal errors (four parameter model)

\begin{tabular}{|c|c|c|c|c|}
\hline$N=30$ & JR & LME & CT & WW \\
\hline Null & 0.0452 & 0.0668 & 0.0474 & 0.0343 \\
\hline LC (Power) & 0.9729 & 0.9911 & 0.9841 & 0.9581 \\
\hline
\end{tabular}

Comment: We recommend CT and JR because WW is too conservative while LME is liberal.

Table 4. Summary table for the power $(\mathrm{SC}=1.5)$ with normal errors (four parameter model)

\begin{tabular}{|c|c|c|c|c|}
\hline$N=30$ & JR & LME & CT & WW \\
\hline Null & 0.0423 & 0.0667 & 0.1023 & 0.0778 \\
\hline SC (Power) & 0.9932 & 1.0 & 1.0 & 0.9993 \\
\hline
\end{tabular}

Comment: We recommend JR at this level of SC because CT, WW, and LME are liberal. It could be clearly seen from the above table that the WW estimates were a little conservative at normal data while the LME is slightly liberal with normal data. They all appear to be valid for the given design matrix. JR is recommended for $\mathrm{SC}=1.5$ while both $\mathrm{JR}$ and $\mathrm{CT}$ are good for $\mathrm{LC}=8$. 


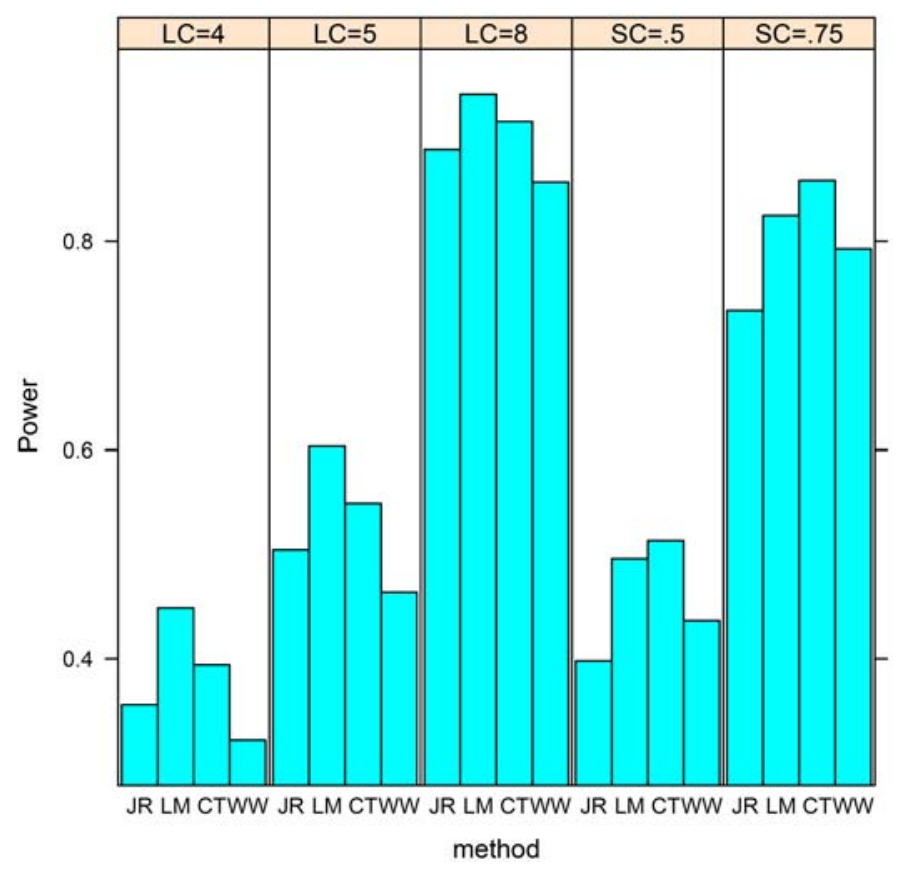

Figure 1. Graph showing the power with normal errors.

Comment: We conclude from the above figure that all methods have good powers when $\mathrm{LC}=8$ and $\mathrm{SC}=0.75$. Thus these points are recommended for use in simulations with normal errors.

Table 5. Summary table for the empirical alpha level with normal errors (four parameter model)

\begin{tabular}{|c|c|c|c|c|}
\hline$N=30$ & JR & LME & CT & WW \\
\hline LC (Null) & 0.0473 & 0.0703 & 0.0493 & 0.037 \\
\hline SC (Null) & 0.0441 & 0.0669 & 0.0471 & 0.0341 \\
\hline
\end{tabular}

Comment: We can see that WW is conservative while LME is liberal. We recommend JR and CT. 
Table 6. Summary table for the power $(\mathrm{LC}=4)$ with normal errors (four parameter model)

\begin{tabular}{|c|c|c|c|c|}
\hline$N=30$ & JR & LME & CT & WW \\
\hline Null & 0.0445 & 0.063 & 0.0452 & 0.0335 \\
\hline LC (Power) & 0.3559 & 0.4487 & 0.3942 & 0.3219 \\
\hline
\end{tabular}

Comment: We can see that WW is conservative while LME is liberal at this level of LC. We recommend JR and CT.

Table 7. Summary table for the power $(\mathrm{SC}=0.75)$ with normal errors (four parameter model)

\begin{tabular}{|c|c|c|c|c|}
\hline$N=30$ & JR & LME & CT & WW \\
\hline Null & 0.0465 & 0.0684 & 0.0876 & 0.0657 \\
\hline SC (Power) & 0.7335 & 0.8246 & 0.8582 & 0.7927 \\
\hline
\end{tabular}

Comment: We recommend JR at this level of SC because CT, WW, and LME are liberal.

Table 8. Summary table for the power $(\mathrm{LC}=5)$ with normal errors (four parameter model)

\begin{tabular}{|c|c|c|c|c|}
\hline$N=30$ & JR & LME & CT & WW \\
\hline Null & 0.0436 & 0.0671 & 0.0479 & 0.0343 \\
\hline LC (Power) & 0.5046 & 0.6035 & 0.549 & 0.4638 \\
\hline
\end{tabular}

Comment: We can see that WW is conservative while LME is liberal at this level of LC. We recommend JR and CT.

Table 9. Summary table for the power $(\mathrm{SC}=0.5)$ with normal errors (four parameter model)

\begin{tabular}{|c|c|c|c|c|}
\hline$N=30$ & JR & LME & CT & WW \\
\hline Null & 0.0439 & 0.0683 & 0.0744 & 0.0545 \\
\hline SC (Power) & 0.398 & 0.4961 & 0.5134 & 0.4367 \\
\hline
\end{tabular}

Comment: We recommend JR and WW at this level of SC because CT and LME are liberal. 
Table 10. Summary table for the power $(\mathrm{LC}=8)$ with normal errors (four parameter model)

\begin{tabular}{|c|c|c|c|c|}
\hline$N=30$ & JR & LME & CT & WW \\
\hline Null & 0.043 & 0.0668 & 0.0471 & 0.0328 \\
\hline LC (Power) & 0.8879 & 0.9409 & 0.9147 & 0.8567 \\
\hline
\end{tabular}

Comment: We can see that WW is conservative while LME is liberal at this level of LC. We recommend JR and CT.

\subsection{Cases of contaminated normal error with 15000 simulations}

Next, we consider contaminated normal errors with the percent of contamination at $20 \%(\& 10 \%)$ and the relative standard deviation at 25 (\& 10), respectively. A simulation study over situations similar to our preliminary study validates the previous results from this analysis.

Table 11. Summary table for the empirical alpha level CN errors $\left(P=0.20, \sigma_{c}=25\right)$

\begin{tabular}{|c|c|c|c|c|}
\hline$N=30$ & JR & LME & CT & WW \\
\hline LC (Null) & 0.0503 & 0.0569 & 0.0481 & 0.0597 \\
\hline SC (Null) & 0.0509 & 0.053 & 0.0435 & 0.0599 \\
\hline
\end{tabular}

Comment: All methods seem good for the empirical alpha level under contaminated normal error setting the level of contamination at $20 \%$ and standard deviation at 25 .

Table 12. Summary table for the empirical alpha level CN errors $\left(P=0.10, \sigma_{c}=10\right)$

\begin{tabular}{|c|c|c|c|c|}
\hline$N=30$ & JR & LME & CT & WW \\
\hline LC (Null) & 0.0468 & 0.0667 & 0.0481 & 0.0578 \\
\hline SC (Null) & 0.0484 & 0.0647 & 0.0461 & 0.0597 \\
\hline
\end{tabular}

Comment: We can see that LME is liberal at this level of contamination but JR, CT, and WW look good. 
Table 13. Summary table for the power $(\mathrm{LC}=5)$ with $\mathrm{CN}$ errors $\left(P=0.20, \sigma_{c}=25\right)$

\begin{tabular}{|c|c|c|c|c|}
\hline$N=30$ & JR & LME & CT & WW \\
\hline Null & 0.0507 & 0.055 & 0.0432 & 0.0609 \\
\hline LC (Power) & 0.3315 & 0.1179 & 0.1345 & 0.4081 \\
\hline
\end{tabular}

Comment: Clearly, WW is liberal at this level of contamination. LME and CT corr test have very low power, thus we recommend JR.

Table 14. Summary table for the power $(\mathrm{SC}=0.75)$ with $\mathrm{CN}$ errors $\left(P=0.20, \sigma_{c}=25\right)$

\begin{tabular}{|c|c|c|c|c|}
\hline$N=30$ & JR & LME & CT & WW \\
\hline Null & 0.0497 & 0.0562 & 0.0475 & 0.0606 \\
\hline SC (Power) & 0.5013 & 0.1563 & 0.1809 & 0.5712 \\
\hline
\end{tabular}

Comment: Clearly, WW is liberal at this level of contamination. LME and CT have very low power, thus we recommend JR.

Table 15. Summary table for the power $(\mathrm{LC}=5)$ with $\mathrm{CN}$ errors $\left(P=0.10, \sigma_{c}=10\right)$

\begin{tabular}{|c|c|c|c|c|}
\hline$N=30$ & JR & LME & CT & WW \\
\hline Null & 0.045 & 0.0687 & 0.0484 & 0.0581 \\
\hline LC (Power) & 0.4557 & 0.4141 & 0.4092 & 0.5118 \\
\hline
\end{tabular}

Comment: Clearly, LME is liberal at this level of contamination. WW, CT corr test and JR have moderate power.

Table 16. Summary table for the power $(\mathrm{SC}=0.75)$ with $\mathrm{CN}$ errors $\left(P=0.10, \sigma_{c}=10\right)$

\begin{tabular}{|c|c|c|c|c|}
\hline$N=30$ & JR & LME & CT & WW \\
\hline Null & 0.0456 & 0.0653 & 0.0664 & 0.0767 \\
\hline SC (Power) & 0.6776 & 0.6063 & 0.6322 & 0.7554 \\
\hline
\end{tabular}


Comment: Clearly, LME, CT, and WW are all liberal at this level of contamination. JR is hereby recommended.

Table 17. Summary table for the power $(\mathrm{LC}=12)$ with $\mathrm{CN}$ errors $\left(P=0.20, \sigma_{c}=25\right)$

\begin{tabular}{|c|c|c|c|c|}
\hline$N=30$ & JR & LME & CT & WW \\
\hline Null & 0.0516 & 0.0579 & 0.0455 & 0.0604 \\
\hline LC (Power) & 0.9497 & 0.4009 & 0.4407 & 0.9153 \\
\hline
\end{tabular}

Comment: All methods are good with $\mathrm{CN}$ errors for $\mathrm{LC}=12, P=0.20$, $\sigma_{c}=25$. JR and WW have better power than LME and CT, but WW is liberal with empirical alpha. JR is recommended.

Table 18. Summary table for the power $(\mathrm{LC}=12)$ with $\mathrm{CN}$ errors $\left(P=0.10, \sigma_{c}=10\right)$

\begin{tabular}{|c|c|c|c|c|}
\hline$N=30$ & JR & LME & CT & WW \\
\hline Null & 0.0471 & 0.0675 & 0.0444 & 0.0592 \\
\hline LC (Power) & 0.9917 & 0.9723 & 0.9297 & 0.9849 \\
\hline
\end{tabular}

Comment: All methods have good power with $\mathrm{CN}$ errors for $\mathrm{LC}=12$, $P=0.10, \sigma_{c}=10$, although LME is liberal with empirical alpha.

Table 19. Summary table for the power $(L C=8)$ with $C N$ errors $\left(P=0.10, \sigma_{c}=10\right)$

\begin{tabular}{|c|c|c|c|c|}
\hline$N=30$ & JR & LME & CT & WW \\
\hline Null & 0.0453 & 0.0655 & 0.0445 & 0.0565 \\
\hline LC (Power) & 0.8436 & 0.7699 & 0.7391 & 0.8513 \\
\hline
\end{tabular}

Comment: All methods have good power with $\mathrm{CN}$ errors for $\mathrm{LC}=8$, $P=0.10, \sigma_{c}=10$, although LME is liberal with empirical alpha. 
Table 20. Summary table for the power $(\mathrm{LC}=8)$ with $\mathrm{CN}$ errors $\left(P=0.20, \sigma_{c}=25\right)$

\begin{tabular}{|c|c|c|c|c|}
\hline$N=30$ & JR & LME & CT & WW \\
\hline Null & 0.0552 & 0.0567 & 0.0449 & 0.0642 \\
\hline LC (Power) & 0.6798 & 0.2133 & 0.2523 & 0.7109 \\
\hline
\end{tabular}

Comment: JR is recommended because WW is liberal while LME and CT have low powers with $\mathrm{CN}$ errors for $\mathrm{LC}=8, P=0.10, \sigma_{c}=10$.

Table 21. Summary table for the power $(\mathrm{SC}=0.6)$ with $\mathrm{CN}$ errors $\left(P=0.10, \sigma_{c}=10\right)$

\begin{tabular}{|c|c|c|c|c|}
\hline$N=30$ & JR & LME & CT & WW \\
\hline Null & 0.0473 & 0.0652 & 0.0589 & 0.0723 \\
\hline SC (Power) & 0.4911 & 0.4496 & 0.4803 & 0.5847 \\
\hline
\end{tabular}

Comment: All methods have moderate power with $\mathrm{CN}$ errors for $\mathrm{SC}=0.6$, $P=0.10, \sigma_{c}=10$. Note that LME and WW are liberal with empirical alpha, we recommend JR.

Table 22. Summary table for the power $(\mathrm{SC}=0.6)$ with $\mathrm{CN}$ errors $\left(P=0.20, \sigma_{c}=25\right)$

\begin{tabular}{|c|c|c|c|c|}
\hline$N=30$ & JR & LME & CT & WW \\
\hline Null & 0.0503 & 0.0548 & 0.0469 & 0.0591 \\
\hline SC (Power) & 0.358 & 0.1217 & 0.1354 & 0.432 \\
\hline
\end{tabular}

Comment: JR and WW have moderate power with $\mathrm{CN}$ errors for $\mathrm{SC}=0.6$, $P=0.20, \sigma_{c}=25$, while LME and CT are not recommended for use at this level of contamination. 


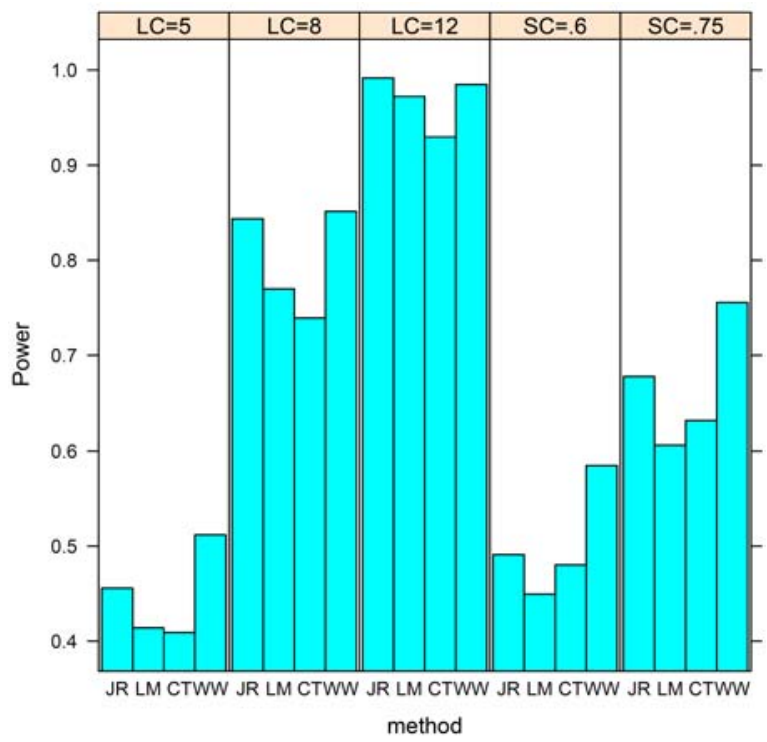

Figure 2. Graph showing the power with contaminated normal errors $P=0.10, \sigma_{c}=10$.

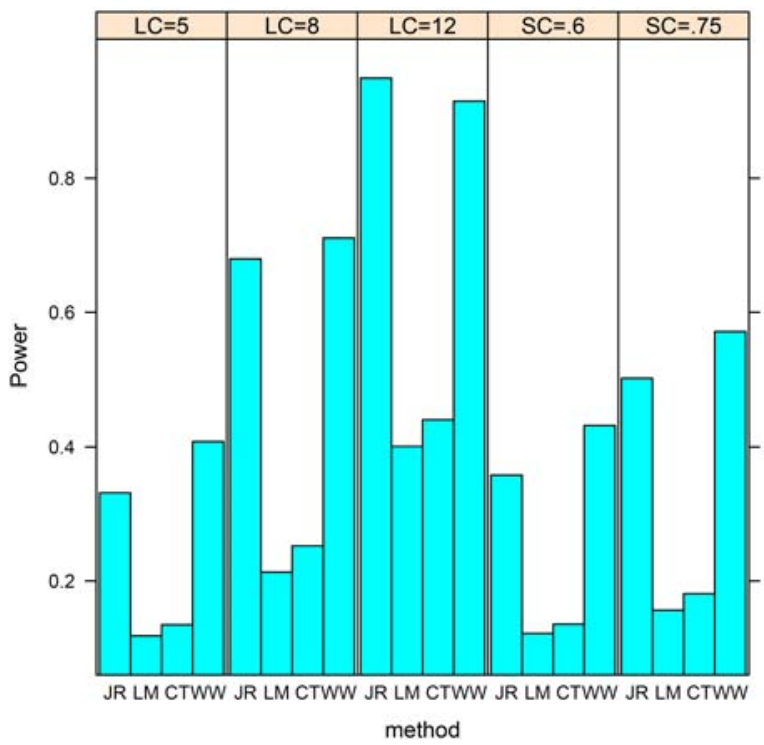

Figure 3. Graph showing the power with contaminated normal errors $P=0.20, \sigma_{c}=25$. 
Table 23. Summary table for the power $(\mathrm{SC}=3.6)$ with $\mathrm{CN}$ errors $\left(P=0.20, \sigma_{c}=25\right)$

\begin{tabular}{|c|c|c|c|c|}
\hline$N=30$ & JR & LME & CT & WW \\
\hline Null & 0.0477 & 0.058 & 0.0565 & 0.0675 \\
\hline SC (Power) & 1.0 & 0.9771 & 0.9529 & 0.9995 \\
\hline
\end{tabular}

Comment: All methods have good power at this value of $\mathrm{SC}$ with $\mathrm{CN}$ errors for $\mathrm{SC}=3.6, P=0.20, \sigma_{c}=25$, but $\mathrm{WW}$ is liberal with empirical alpha.

\subsection{Cases of Cauchy error with 15000 simulations}

Table 24. Summary table for the empirical alpha level with cauchy errors

\begin{tabular}{|l|l|l|l|l|}
\hline$N=30$ & JR & LME & CT & WW \\
\hline LC (Null) & 0.0523 & 0.0598 & 0.0471 & 0.0537 \\
\hline SC (Null) & 0.0538 & 0.0435 & 0.0435 & 0.0519 \\
\hline
\end{tabular}

Comment: All methods look good for the empirical alpha level.

Table 25. Summary table for the power $(\mathrm{LC}=5)$ with Cauchy errors

\begin{tabular}{|c|c|c|c|c|}
\hline$N=30$ & JR & LME & CT & WW \\
\hline Null & 0.0558 & 0.0467 & 0.0432 & 0.0531 \\
\hline LC (Power) & 0.2467 & 0.0839 & 0.1237 & 0.2691 \\
\hline
\end{tabular}

Comment: All the methods show poor power performance with Cauchy Errors for this level of LC.

Table 26. Summary table for the power $(\mathrm{SC}=0.75)$ with Cauchy errors

\begin{tabular}{|c|c|c|c|c|}
\hline$N=30$ & JR & LME & CT & WW \\
\hline Null & 0.0549 & 0.0547 & 0.0463 & 0.0531 \\
\hline SC (Power) & 0.3711 & 0.0977 & 0.1712 & 0.4029 \\
\hline
\end{tabular}

Comment: JR and WW have moderate power with Cauchy errors for $\mathrm{SC}=0.75$, but LME and CT have poor power. 
Table 27. Summary table for the power study $(\mathrm{LC}=12)$ with Cauchy errors

\begin{tabular}{|c|c|c|c|c|}
\hline$N=30$ & JR & LME & CT & WW \\
\hline Null & 0.0537 & 0.0454 & 0.0423 & 0.0517 \\
\hline LC (Power) & 0.8465 & 0.2188 & 0.3684 & 0.8214 \\
\hline
\end{tabular}

Comment: JR and WW have good power with Cauchy errors for $\mathrm{LC}=12$, but LME and CT are not so good.

Table 28. Summary table for the power study $(\mathrm{LC}=8)$ with Cauchy errors

\begin{tabular}{|c|c|c|c|c|}
\hline$N=30$ & JR & LME & CT & WW \\
\hline Null & 0.0544 & 0.0457 & 0.0403 & 0.049 \\
\hline LC (Power) & 0.5126 & 0.1285 & 0.2312 & 0.537 \\
\hline
\end{tabular}

Comment: JR and WW have good power with Cauchy errors for $\mathrm{LC}=12$, but LME and CT are not good.

Table 29. Summary table for the power study $(\mathrm{SC}=0.6)$ with Cauchy errors

\begin{tabular}{|c|c|c|c|c|}
\hline$N=30$ & JR & LME & CT & WW \\
\hline Null & 0.0555 & 0.0554 & 0.048 & 0.0546 \\
\hline SC (Power) & 0.2658 & 0.0768 & 0.1293 & 0.2935 \\
\hline
\end{tabular}

Comment: All methods have poor power at this value of $\mathrm{SC}=0.6$ with Cauchy errors. 


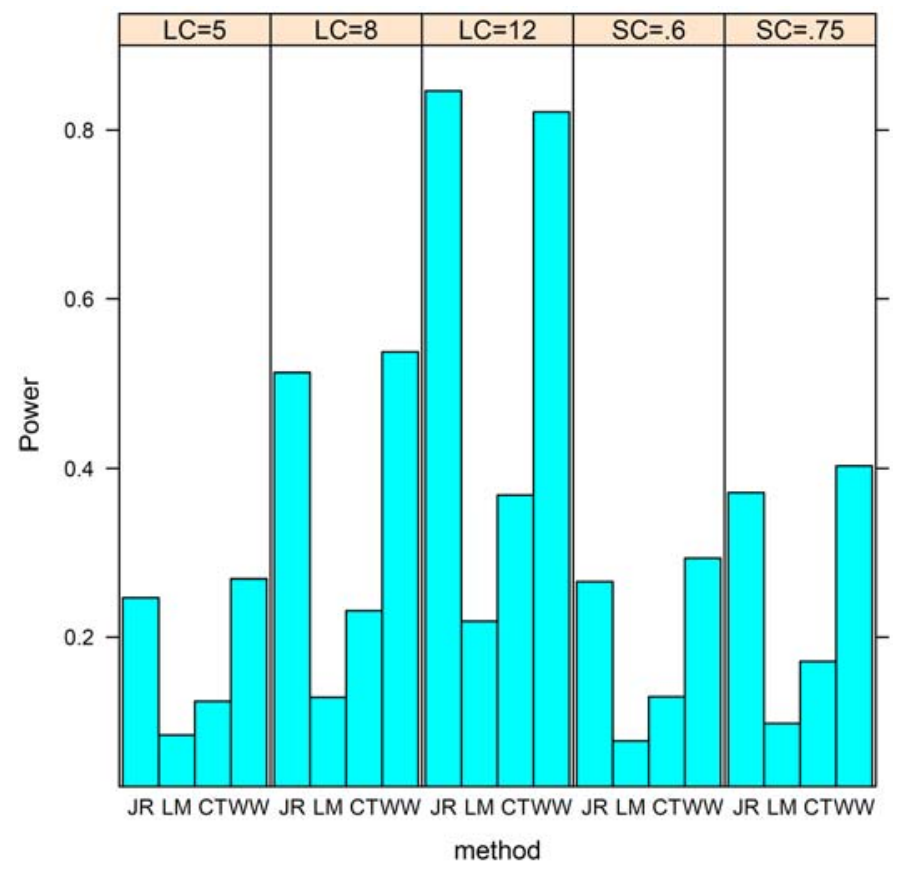

Figure 4. Graph showing the power with Cauchy errors.

\subsection{Cases of contaminated skewed Cauchy error with 15000 simulations}

Table 30. Summary table for the empirical alpha level with contaminated skewed Cauchy errors $\left(P=0.20, \sigma_{c}=25\right)$

\begin{tabular}{|c|c|c|c|c|}
\hline$N=30$ & JR & LME & CT & WW \\
\hline LC (Null) & 0.0491 & 0.0497 & 0.0464 & 0.0249 \\
\hline SC (Null) & 0.0567 & 0.0501 & 0.0438 & 0.0237 \\
\hline
\end{tabular}

Comment: All methods except WW have good empirical alpha level with contaminated skewed Cauchy errors but WW is conservative. 
Table 31. Summary for the power $(\mathrm{LC}=5)$ with contaminated skewed Cauchy errors $\left(P=0.20, \sigma_{c}=25\right)$

\begin{tabular}{|c|c|c|c|c|}
\hline$N=30$ & JR & LME & Corr test & WW \\
\hline Null & 0.0541 & 0.0543 & 0.0451 & 0.024 \\
\hline LC (Power) & 0.1483 & 0.0566 & 0.0583 & 0.1473 \\
\hline
\end{tabular}

Comment: All the methods perform poorly with contaminated skewed Cauchy errors for $\mathrm{LC}=5$. We need to increase the starting value of the LC.

Table 32. Summary for the power $(\mathrm{SC}=0.75)$ with contaminated skewed Cauchy errors $\left(P=0.20, \sigma_{c}=25\right)$

\begin{tabular}{|c|c|c|c|c|}
\hline$N=30$ & JR & LME & Corr test & WW \\
\hline Null & 0.0471 & 0.0519 & 0.0497 & 0.0259 \\
\hline SC (Power) & 0.2438 & 0.0637 & 0.0627 & 0.2318 \\
\hline
\end{tabular}

Comment: All the methods perform poorly with contaminated skewed Cauchy errors for $\mathrm{SC}=0.75$.

Table 33. Summary for the power $(\mathrm{LC}=12)$ with contaminated skewed Cauchy errors $\left(P=0.20, \sigma_{c}=25\right)$

\begin{tabular}{|c|c|c|c|c|}
\hline$N=30$ & JR & LME & Corr test & WW \\
\hline Null & 0.0575 & 0.0539 & 0.0489 & 0.0233 \\
\hline LC (Power) & 0.6703 & 0.0949 & 0.1107 & 0.52 \\
\hline
\end{tabular}

Comment: JR has good power with contaminated skewed Cauchy errors for $\mathrm{LC}=12$, but $\mathrm{LME}$ and $\mathrm{CT}$ have very low power. $\mathrm{WW}$ is too conservative thus we recommend JR.

Table 34. Summary for the power $(\mathrm{SC}=0.6)$ with contaminated skewed Cauchy errors $\left(P=0.20, \sigma_{c}=25\right)$

\begin{tabular}{|c|c|c|c|c|}
\hline$N=30$ & JR & LME & Corr test & WW \\
\hline Null & 0.0476 & 0.0496 & 0.0474 & 0.0242 \\
\hline SC (Power) & 0.1679 & 0.0573 & 0.0569 & 0.1663 \\
\hline
\end{tabular}


Comment: All the methods perform poorly with contaminated skewed Cauchy errors for $\mathrm{SC}=0.6$.

Table 35. Summary for the power $(\mathrm{SC}=3.6)$ with contaminated skewed Cauchy errors $\left(P=0.20, \sigma_{c}=25\right)$

\begin{tabular}{|c|c|c|c|c|}
\hline$N=30$ & JR & LME & Corr test & WW \\
\hline Null & 0.05 & 0.0528 & 0.0529 & 0.0273 \\
\hline SC (Power) & 0.9913 & 0.336 & 0.3669 & 0.9435 \\
\hline
\end{tabular}

Comment: JR has good power with contaminated skewed Cauchy errors when $\mathrm{SC}=3.6, P=0.20, \sigma_{c}=25$, but LME and CT are not good. We recommend JR. WW is extremely conservative with empirical alpha.

Table 36. Summary for the power $(\mathrm{SC}=2.0)$ with contaminated skewed Cauchy errors $\left(P=0.20, \sigma_{c}=25\right)$

\begin{tabular}{|c|c|c|c|c|}
\hline$N=30$ & JR & LME & Corr test & WW \\
\hline Null & 0.0488 & 0.0505 & 0.0465 & 0.0261 \\
\hline SC (Power) & 0.909 & 0.1392 & 0.16 & 0.7111 \\
\hline
\end{tabular}

Comment: JR has good power with skew Cauchy contaminated errors when $\mathrm{SC}=2.0, P=0.20, \sigma_{c}=25$, but LME and CT are not good. We recommend JR. WW is extremely conservative with empirical alpha. 


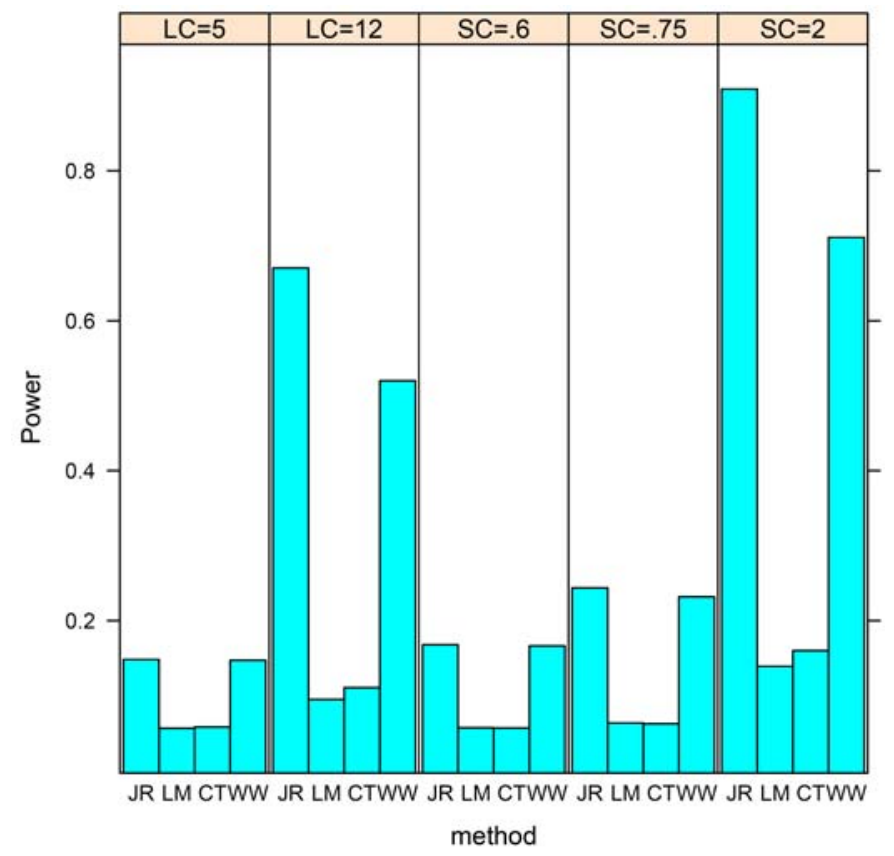

Figure 5. Graph showing the power with contaminated skewed Cauchy errors $P=0.20, \sigma_{c}=25$.

Clearly, for simulations based on case of independence within series, but dependence between two or more baselines, it can be seen that JR is more consistent, efficient and even superior compared to the results obtained from the other methods when the errors were contaminated for both normal and Cauchy. For different values of LC and SC, LME and CT were greatly distorted by the level of contamination introduced (added) into the simulation. The effect of these contamination on the LME and CT might lead to a wrong conclusion and hence, render the purpose of an experiment worthless. Also, we observed that JR compete favorably with the correlation test under the case of normal and WW even perform better than JR with Cauchy error.

In furtherance of this work, we employed the concept of a generalized inverse to obtain the variance-covariance structure for the JR estimates and the LME model. A corresponding meta analysis component that 
resulted from both the JR estimates and the LME model, respectively, were compared with the result from the "CT" via empirical type I error and power study of these tests. We illustrate our meta procedure in the following example.

\section{Examples on JR, LME, and CT}

\subsection{Example 1}

We use our simulation code to generate sample data based on normal errors and contaminated normal errors, respectively. There are 3 baseline series in each simulation using sample sizes $12,20,30$, and 50, respectively. This is simulated data so we know the answers which procedures were effective and which were not. The meta analyses table obtained from the output are summarized below:

Table 37. Meta analysis on JR, LME, and CT using normal errors

\begin{tabular}{|c|c|c|c|c|c|c|c|c|c|c|c|c|}
\hline & \multicolumn{10}{|c|}{$n$} \\
\hline & \multicolumn{3}{|c|}{12} & \multicolumn{3}{c|}{20} & \multicolumn{3}{c|}{30} & \multicolumn{3}{c|}{50} \\
\hline Stat & JR & LME & CT & JR & LME & CT & JR & LME & CT & JR & LME & CT \\
\hline LC & 0.58 & 0.25 & 0.50 & 0.24 & -0.11 & 0.68 & 0.32 & 0.27 & -0.05 & -1.22 & -1.3 & -0.10 \\
\hline$t$ & 0.89 & 0.50 & 1.40 & 0.27 & -0.12 & 1.32 & 0.45 & 0.41 & -0.12 & -1.94 & -2.3 & -0.30 \\
\hline pval & 0.40 & 0.63 & 0.14 & 0.79 & 0.91 & 0.16 & 0.66 & 0.69 & 0.39 & 0.09 & 0.05 & 0.38 \\
\hline
\end{tabular}

The CT procedure is more effective than other methods with normal errors but the JR procedure seems more consistent while LME does not look good because of its fluctuation.

Clearly, it can be seen that JR is more consistent, efficient and even superior when compared with the results obtained from the other methods using contaminated normal errors. 
Table 38. Meta analysis on JR, LME, and CT using contaminated normal errors

\begin{tabular}{|c|c|c|c|c|c|c|c|c|c|c|c|c|}
\hline & \multicolumn{10}{|c|}{$n$} \\
\hline & \multicolumn{3}{|c|}{12} & \multicolumn{3}{c|}{20} & \multicolumn{3}{c|}{30} & \multicolumn{3}{c|}{50} \\
\hline Stat & JR & LME & CT & JR & LME & CT & JR & LME & CT & JR & LME & CT \\
\hline LC & 2.08 & 6.11 & 3.41 & 1.37 & 3.0 & 0.76 & -0.89 & -0.84 & 0.50 & -0.11 & -1.6 & -0.54 \\
\hline$t$ & 0.90 & 1.81 & 1.84 & 1.23 & 0.93 & 0.87 & -0.93 & -0.35 & 0.02 & -0.17 & -0.79 & -0.55 \\
\hline pval & 0.39 & 0.11 & 0.08 & 0.25 & 0.38 & 0.26 & 0.38 & 0.74 & 0.40 & 0.87 & 0.45 & 0.34 \\
\hline
\end{tabular}

\subsection{Example 2}

A ficticious data set having intervention introduced mid-way in each series of size 20 is being considered here. We introduced (added) some outliers into this ficticious data to see the effect on the result obtained via each of these methods. The output is outlined in the following table:

Table 39. Meta analysis on JR, LME, and CT using data with and without outliers

\begin{tabular}{|c|r|r|r|r|r|r|}
\hline & \multicolumn{6}{|c|}{ Methods } \\
\hline & \multicolumn{2}{|c|}{ Without outliers } & \multicolumn{3}{|c|}{ With outliers } \\
\hline Stat & JR & LME & CT & JR & LME & CT \\
\hline LC & -2.00 & -3.301 & 0.0625 & -3.25 & -860584.5 & -9657.34 \\
\hline$t$ & -0.8393 & -1.2223 & -0.0271 & -1.0357 & -0.6934 & -0.8418 \\
\hline pval & 0.4256 & 0.2564 & 0.3917 & 0.3306 & 0.5077 & 0.2706 \\
\hline
\end{tabular}

Clearly, it can be seen that JR is more consistent, efficient and even superior compared to the results obtained from the other methods when outliers were introduced into the series. The level change estimate from LME and CT were greatly distorted by the outliers. The effect of these outliers on the LME and CT might lead to a wrong conclusion and hence, render the purpose of an experiment worthless. 


\section{Conclusion}

This study creates a new parametric method, and a new robust approach, to obtain weighted statistical components of multiple baseline time-series design intervention models. The parametric procedures are based on least squares (LS) estimations. The robust procedures are similar to the parametric procedures, in that, instead of LS estimates, robust estimates of the parameters were used. Also, we did a robust HMZ diagnostic used to check dependency within series. A Monte Carlo evaluation of these methods are provided. These include comparison between parametric and robust procedures.

The test results of these new approaches were compared with a joint rank (JR) based estimation and linear mixed effect (LME) model as discussed under simulation results. The new parametric approach shows a better consistency and good power than the robust analogue for the case of normal errors. The new robust approach is more conservative but has a better power than the new parametric method for the case of contaminated errors. The JR method seems more appropriate in term of its empirical type I error and the power of the test for the case of independence within but dependence between series than other methods (i.e., CT, WW, and LME) considered in this study.

We illustrated the robustness of the procedures on a real data set which contained some outliers. Our robust procedures were much less sensitive to the effect of the outliers than the traditional analysis based on LS. A simulation study over situations similar to that of the data set confirmed the validity of our new approaches. The study also showed the robustness of efficiency of our approach over that of the traditional analysis. 


\section{References}

[1] O. A. Awosoga, Meta Analyses of Multiple Baseline Time Series Design Intervention Models for Dependent and Independent Series, Unpublished PhD Dissertation, Western Michigan University, 2009.

[2] L. V. Hedges and I. Olkin, Nonparametric estimators of effect size in meta-analysis, Psychological Bulletin 96 (1984), 573-580.

[3] T. P. Hettmansperger and J. W. McKean, Robust Nonparametric Statistical Methods, Kendalls Library of Statistics 5, Great Britain, Arnold, 1998.

[4] T. P. Hettmansperger, J. W. McKean and S. J. Sheather, Robust nonparametric methods, Journal of the American Statistical Association 95 (2000), 1308-1312.

[5] B. E. Huitema, Anaysis of interrupted time-series experiments using ITSE: A critique, Understanding Statistics: Statistical Issues in Psychology, Education, \& the Social Sciences 3 (2004b), 27-46.

[6] B. E. Huitema and J. W. McKean, Irrelevant autocorrelation in least-squares intervention models, Psychological Methods 3 (1998), 104-116.

[7] B. E. Huitema and J. W. McKean, Design specification issues in time-series intervention models, Education \& Psychological Measurement 60 (2000a), 38-58.

[8] B. E. Huitema and J. W. McKean, A simple and powerful test for autocorrelated errors in OLS intervention models, Psychological Reports 87 (2000b), 3-20.

[9] B. E. Huitema, J. W. McKean and S. Laraway, Time-series intervention analysis using ITSACORR: Fatal Flaws, Journal of Modern Applied Statistical Methods (JMASM) 6(2) (2007), 367-379.

[10] D. Kahner, C. Moler and S. Nash, Numerical Methods and Software, Englewood Cliffs, NJ: Prentice Hall, 1988.

[11] A. E. Kazdin, Single - Case Research Designs, Methods for Clinical and Applied Settings, Oxford University Press, Inc., pp. 126-151, 1982.

[12] M. J. Koehler and J. R. Levin, RegRand: Statistical software for the multiplebaseline design, Behavior Research Methods, Instruments \& Computers 32(2) (2000), 367-371.

[13] J. D. Kloke, J. W. McKean and M. M. Rasid, Rank -based estimation and associated inferences for linear models with clustered correlated errors, Journal of the American Statistical Association 104(485) (2009), 384-390.

[14] G. Marsaglia and T. A. Bray, A convenient method for generating normal variables, Society of Industrial and Applied Mathematics 6(3) (1986), 260-264.

[15] J. W. McKean, Robust analyses of linear models, Journal of Statistical Science 19(4) (2004), 562-570.

[16] Y. Sakamoto, M. Ishiguro and G. Kitagawa, Akaike Information Criterion Statistics, D. Reidel Publishing Company, Dordrecht/Tokyo, 1986. 
[17] G. Schwarz, Estimating the dimension of a model, The Annals of Statistics 6(2) (1978), 461-464. Published by: Institute of Mathematical Statistics.

[18] W. R. Shadish, T. D. Cook and D. T. Campbell, Experimental and Quasi Experimental Designs for Generalized Causal Inference, Boston: Houghton-Mifflin, 2002.

[19] J. T. Terpstra and J. W. McKean, Rank-based analyses of linear models using R, Journal of Statistical Software 14(7) (2005).

[20] F. M. Wolf, Meta-Analysis: Quantitative Methods for Research Synthesis, (Quantitative Applications in the Social Sciences 59) SAGE Publications, Inc. Beverly Hills, California, 1986. 


\section{Appendix A}

\section{H-M Design Matrix}

Form of data entry for regression analysis of two-phase (4-parameter) time-series intervention model

Table C.1. General form of two-phase (4-parameter) data entry

\begin{tabular}{cccc}
\hline $\begin{array}{c}\text { Measurement Occasion } \\
\text { Variable T }\end{array}$ & $\begin{array}{c}\text { Dummy } \\
\text { Variable D }\end{array}$ & $\begin{array}{c}\text { SC Variable } \\
{\left[T-\left(n_{1}+1\right)\right] D}\end{array}$ & $\begin{array}{c}\text { Dependent } \\
\text { Variable Y }\end{array}$ \\
\hline 1 & 0 & 0 & $Y_{1}$ \\
2 & 0 & 0 & $Y_{2}$ \\
3 & $\cdot$ & $\cdot$ & $\cdot$ \\
$\cdot$ & $\cdot$ & $\cdot$ & $\cdot$ \\
$\cdot$ & $\cdot$ & $\cdot$ & $\cdot$ \\
$n_{1}$ & 0 & 0 & $Y_{n_{1}}$ \\
\hline$n_{1}+1$ & 1 & 0 & $Y_{n_{1}+1}$ \\
$n_{1}+2$ & 1 & 1 & $Y_{n_{1}+2}$ \\
$n_{1}+3$ & 1 & 2 & $Y_{n_{1}+3}$ \\
$\cdot$ & $\cdot$ & $\cdot$ & $\cdot$ \\
$\cdot$ & $\cdot$ & $\cdot$ & $\cdot$ \\
$n_{1}+n_{2}=N$ & 1 & $n_{2}-1$ & $Y_{n_{1}+n_{2}}=Y_{n}$ \\
\hline
\end{tabular}

$n_{1}$ and $n_{2}$ are the number of observations in phases 1 and 2, respectively. (AB Design) As earlier mentioned, our new parametric and robust approach can be extended to more than two phase designs. See Awosoga [1] for an appropriate design matrices for these extensions. 